\title{
Animating formal specifications with inheritance in a DL-based framework ${ }^{1}$
}

\author{
Pedro Sánchez Patricio Letelier Isidro Ramos \\ Department of Information Systems and Computation \\ Valencia University of Technology \\ Camino de Vera, s/n, 46071 Valencia \\ email \{ppalma | letelier | iramos\}@dsic.upv.es \\ http://www.dsic.upv.es/users/oom
}

Keywords: Requirements engineering, object oriented methods, inheritance, animation of specifications.

\begin{abstract}
Dynamic Logic (DL) provides a suitable formal framework to model actions and reasoning about them. OASIS is a language for the specification of object oriented conceptual models. In our model, specialization is a relation between classes that defines an inheritance mechanism through static and dynamic partitions. A variant of DL (including the Deontic operators for permission, prohibition and obligation) is the formalism used in OASIS to deal with changes of state, triggers, preconditions, protocols and operations. The animation of conceptual models in order to validate the specification is an interesting topic. We have worked on translating OASIS specifications automatically to concurrent environments in order to obtain a prototype useful to validate specifications by animation. The aim of this paper is to show that it is feasible to translate static and dynamic partitions automatically into dynamic logic formulae. Thus, using the same developed schema of animation it is possible to execute OASIS specifications including inheritance.
\end{abstract}

\section{Introduction}

Conceptual models, representing the functional requirements of information systems, are a key factor when linking problem and solution domains. Building a conceptual model is a discovery process, not only for analysts but also for stakeholders. The most suitable strategy in this situation is to build conceptual models in an iterative and incremental way, through analyst and stakeholder interaction. Conceptual modeling involves four activities: elicitation of requirements, modeling or specification, verification of quality and consistency, and eventually, validation.

\footnotetext{
${ }^{1}$ This research is supported by the "Comisión Interministerial de Ciencia y Tecnología" (CICYT) through the MENHIR proyect (grant no. TIC97-0593-C05-01).
} 
Formal methods for conceptual modeling provide improvements in soundness and precision for specifications and help in their verification. However, when considering elicitation and requirements validation, prototyping techniques are more often used. Hence, it is interesting to obtain a combination of both approaches.

This work uses OASIS [10,7] (Open and Active Specification of Information Systems), a formal approach for object oriented conceptual specification of information systems. This is a step forward in a growing research field where the validation of formal specifications through animation is being explored [16]. In this sense, some other proposals close in nature to $O A S I S$ and to the work we are carrying out using OASIS are TROLL [5], ALBERT [6] and OBLOG [13]. The differences between these works and ours are basically determined by features of the underlying formalisms and the offered expressiveness. According to the presented results, the state of art is similar and is characterized by preliminary versions of animation environments.

Validation and verification play an important role in the quality of the final product. By means of validating formal specifications stakeholders can fasten the correspondence between formal specifications and user prospects. The verification process allows to verifying the correctness between implementations and specification. Errors can be propagated towards the design and implementation process if the validation and/or verification are not done exhaustively. Basically, validation and verification techniques can be classified in two groups:

- Statics: reasoning about system properties regarding a set of predefined rules.

- Dynamics: executing some kind of implementation of the system.

As Feenstra points out in [1] two approaches are considered when animating specifications: to reason about scenarios (what requires an automatically generated prototype obtained from formal specifications) and to reason about reachability properties (what requires software capable of solving queries automatically). In our work the animation process focuses on the first approach showing the effects of actions in the system by testing scenarios.

Figure 1 shows a framework based on OASIS for elicitation, modeling, verification and validation of requirements. Elicitation is achieved by using scenarios [14]. The elements and expected behavior of a given specification are extracted by analysts from scenarios. Functional requirements are modeled using a graphical specification module based on $O A S I S$. Conceptual models can be verified according to OASIS formal properties. At each stage of the requirements specification process it would be possible to validate the behavior of the associated prototype against the expected behavior. This comparison could lead to updates or extensions of existing scenarios. This cycle continues until the requirements are compliant with the proposed set of scenarios.

Experiments have been carried out using Object-Oriented Petri Nets [15] and Concurrent Logic Programming [8] as concurrent environments for OASIS specifications. Correspondences between OASIS and these environments have been included in a translator program. This translator takes an OASIS specification stored in the repository and generates automatically a Concurrent Logic Program or a Petri Net that constitute a prototype for the corresponding conceptual model. Furthermore, through a preliminary version of the graphical animation environment, the analyst can interact with the prototype in a suitable way. We obtain CodeSign code automatically 
from OASIS specifications. This work is being integrated into a CASE tool for system modeling supporting the OASIS approach. We have addressed validation through animation focusing on OASIS but this work could be extended to other similar languages. Basically, the obtained prototypes allow to animate sets of a variant of Dynamic Logic formulae [12] (including permissions, valuations and obligations) representing OASIS specifications. Our aim is to be able to animate (using the same translator) $O A S I S$ specifications including inheritance.

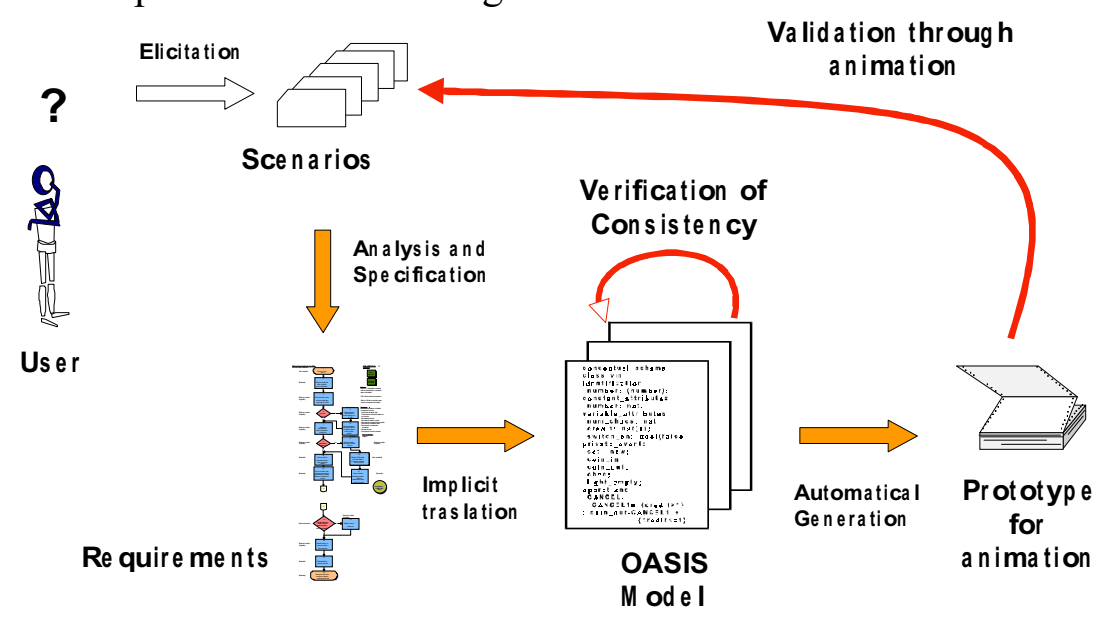

Figure 1: Animation Environment.

Nowadays, an increasing work in validation through animation of conceptual models has been developed. Many CASE tools have integrated an special module for animation. Most of tools related to requirements validation do not offer much help for animation. OBLOG ${ }^{2}$ has an animation module but it is not still available. In Rhapsody ${ }^{3}$ and ObjectTime ${ }^{4}$ a graphical representation (a sequence diagram) is included as a result of the animation and interaction between objects. In this way, Aonix ${ }^{5}$ offers a module called "Animator" in which the edition and execution of scenarios are supported. In BridgePoint ${ }^{6}$ validation is done by monitoring in several levels the generated prototype but it is not easy to contrast between the obtained and expected results. In [3] an environment about TROLL in which is possible to study the change in the state of objects is showed. In a similar way, in [6] the architecture and functionality of an animation module for ALBERT specifications is presented. In all these proposals near to $O A S I S$, the state of the art is clearly characterized by preliminary versions of animation modules. The aim of this paper is to show that is feasible to translate automatically the $O A S I S$ specialization in $D L$ formulae. Thus using the same developed schema of animation it is possible to execute OASIS specifications including inheritance.

\footnotetext{
${ }^{2}$ http://www.oblog.pt

3 http://www.ilogix.com

${ }_{5}^{4}$ http://www.objectime.com

5 http://www.aonix.com

${ }^{6}$ http://www.projtech.com
} 
The rest of this paper is organized as follows: in section 2 we introduce the basic concepts of $O A S I S$. Section 3 gives the formal framework of specialization in OASIS. Section 4 gives a short description of the used $D L$. Section 5 establishes a representation mechanism for specialization. Section 5 summarizes the paper and outlines further work. Finally, an appendix introduces a full case study.

\section{Basic concepts of $O A S I S$}

An $O A S I S$ specification is a presentation of a theory in the used formal system and is expressed as a structured set of class definitions. Classes can be simple or complex. A complex class is defined in terms of other classes (simple or complex) by establishing relationships among classes. These relationships provide aggregation or inheritance mechanisms. A class has a name, one or more identification mechanisms for its instances (objects) and a type or template that is shared by every instance belonging to the class. Each object has an unique identifier (oid) set by the system, however, objects are referred by their identification mechanisms belonging to the problem space. A function establishes a mapping between the identification mechanisms and the oid. The type or template describes the structure and behavior of every object.

Thus each object encapsulates its own state and behavior rules. As usual in object oriented environments, objects can be seen from two points of view: static and dynamic. From the static perspective, the attributes are properties describing the object structure. The object state in a given instant is the set of structural properties values. From the dynamic perspective, the evolution of objects is characterized by the "change of state" notion. The occurrence of actions implies changes (by means of valuations and derivations) in the values of the attributes. Object activity is determined by a set of rules: preconditions (as forbidden actions in certain states), triggers (as obligations to be fulfilled in certain states), protocols (as allowed sequences of actions in object life) and operations (as obligated sequences of actions). A step is the set of actions executed at the same instant by the object.

Inheritance is a mandatory characteristic of the object oriented paradigm. Inheritance is a mechanism through which subclasses inherit properties of superclasses. The inheritance mechanism is an unquestionable help in incremental construction and reuse, but researches rarely agree on its meaning and usage [17]. In OASIS, inheritance is used in a disciplined way under the concept of specialization.

\section{Specialization in $O A S I S$}

In $O A S I S$ to specialize a class means to create one or more partitions for it. Each partition is a set of new classes that divides the original one taking into account some criterions, thus many partitions can coexist. One object, in a given instant, is instance of only one subclass in every partition. Next we give the characteristics of each kind of specialization in $O A S I S$, it is, static partitions and dynamic partitions.

Definition 1 Subclasses and superclasses. When a subclass is a specilization of another class, the former is said to be a subclass and the latter a superclass. The template of a subclass is derived from the templates of other classes (superclasses). 


\subsection{Static partitions}

A static partition [18] divides completely the possible instances set of the partitioned class into disjoints subsets. Thus, static partitions divide the space of objects. Each object is permanently created as an instance of a given subclass in static partitions.

Each instance of a given subclass is at the same time one instance of its superclass and vice versa.

Example 1 Two static partitions of class vehicle:

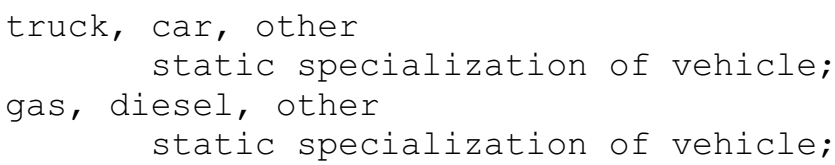

\subsection{Dynamic partitions}

A dynamic partition [18] includes subclasses to which an object can belong during its lifetime. Object migration between classes is produced by the occurrence of actions. Thus, a dynamic partition divides the possible states of objects allowing to objects to change their subclasses.

From the point of view of the OASIS language, we can specify the transition between subclasses in a partition in two manners (being equivalent): by means of a migration process (related to occurring actions) or by attribute values.

Example 2 A dynamic specialization of class car produced by the occurrence of the actions new_car, and repair_car in OASIS:

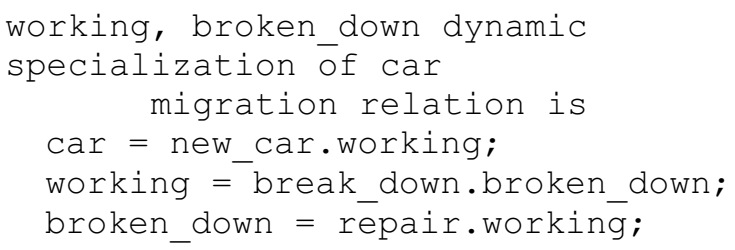

The creation of a car instance implies that it starts belonging to the class working. As an instance of working, actions from car and working templates can be recognized. The action break_down implies to leave the subclass working and to migrate towards broken_down class.

From a theoretical point of view, the process representing the life of an instance of car is the joint of processes defined in every subclass. The connections among subclasses are given by the actions included in the migratory process.

Example 3 A dynamic partition of the class person under the attribute approach:

child where $\{$ age $<13$ \}

teenager where $\{13<=$ age and age $<=19\}$ 
In this case, whenever the attribute age changes, depending on the established conditions one instance of person could migrate between subclasses.

Eventually, in $O A S I S$ is not allowed to define static partitions from dynamic ones.

\subsection{Species and multiple inheritance in $O A S I S$}

The Cartesian product between leaf classes of a given specialization hierarchy gives the set of species mixing all properties of those classes. Furthermore, emergent properties can be specified in their templates.

Example 4 Species of the hierarchy vehicle:

truck*diesel, car*gas, car*diesel

other*diesel, other*gas, truck*gas

Example 5 The class truck*diesel may have an emergent attribute named tonnage. This attribute must be specified in the truck*diesel template.

\section{OASIS formalized in $D L$}

Dynamic logic (DL) [4] has been traditionally considered to describe and reason about dynamic systems. The aim of this logic is the study of mathematical properties of programs and their behaviour. An object in $O A S I S$ is able to pass from one state to another. Due to the state change, the truth values of the formulas describing the state also change. The objective in our work is to define a logical basis to be able to express our reasoning about OASIS specifications. To each OASIS object we associate an accessibility relation in such a way that a pair of state $(s, t)$ is in that relation if and only if there is a computation of the program (execution of a set of actions by the object) transforming the state $s$ into the state $t$.

In [12] Deontic Logic is described as a variant of $D L$ [4]. The definition of deontic operators in $D L$ is:

\begin{tabular}{|c|c|}
\hline $\mathrm{O}(a) \Leftrightarrow[\neg a]$ false & "the occurrence of $a$ is obligatory". \\
\hline $\mathrm{F}(a) \Leftrightarrow[a]$ false & "the occurrence of $a$ is forbidden". \\
\hline \multirow{2}{*}{$\mathrm{P}(a) \Leftrightarrow \neg \mathrm{F}(a)$} & " $a$ is permitted if and only if $a$ \\
\hline & is not forbidden". \\
\hline
\end{tabular}

Where false denotes no reachable world. It is usual to find in related works the special atom V of violation representing a problematic or "non-ideal" situation. In our work the atom false expresses the impossibility of performing a transition. 
We are using a sublanguage of the language presented by Meyer in [12] including the following kind of formulae:

\begin{tabular}{|c|c|}
\hline \multirow{2}{*}{$\psi \rightarrow[a]$ false } & "the occurrence of $a$ is forbidden \\
\hline & in states where $\psi$ is satisfied" \\
\hline \multirow{2}{*}{$\psi \rightarrow[\neg \mathrm{a}]$ false } & "the occurrence of $a$ is obligatory \\
\hline & in states where $\psi$ is satisfied" \\
\hline \multirow{3}{*}{$\psi \rightarrow[\mathrm{a}] \phi$} & "immediately after $a$ occurrence, \\
\hline & $\phi$ must be satisfied \\
\hline & in states where $\psi$ is satisfied" \\
\hline
\end{tabular}

These formulae are named prohibition, obligation and valuation, respectively. Additionally, $\psi$ is a well-formed formula that characterizes the state of an object when the action $a$ occurs and $\neg a$ represents the non-occurrence of the action $a$ (i.e. only other actions different from $a$ could occur). Furthermore, there is no state satisfying the atom false. Thus, one action is forbidden if its occurrence leads the system towards a violation state, and one action is obligatory if its non-occurrence leads the system towards a violation state.

An OASIS class template is represented by the tuple $\langle A t r$, Ev, Formulae, Processes $\rangle$, that is, attributes, events, $D L$ formulae (valuations, prohibitions and obligations) and process specifications (protocols and operations). In [9] it is showed how Processes can also be interpreted as a set of $D L$ formulae. Thus, the class template can be seen as $\langle$ Atr, $E v, D L F o r m\rangle$ where DLForm includes all $D L$ formulae establishing the behavior of objects. Next section details how, through a translation process, static and dynamic partitions can be integrated in the same formal framework of $D L$.

\section{Specialization in $D L$}

As mentioned in previous sections species involve classes in the same hierarchy. Likewise, the classes constituting species have a common ancestor class which has been partitioned more than once. Figure shows a specialization hierarchy with the following species:

$$
\begin{array}{lll}
\mathrm{C}_{3} * \mathrm{C}_{1} & \mathrm{C}_{3} * \mathrm{C}_{6} & \mathrm{C}_{3} * \mathrm{C}_{7} \\
\mathrm{C}_{4} * \mathrm{C}_{1} & \mathrm{C}_{4} * \mathrm{C}_{6} & \mathrm{C}_{4} * \mathrm{C}_{7} \\
\mathrm{C}_{5} * \mathrm{C}_{1} & \mathrm{C}_{5} * \mathrm{C}_{6} & \mathrm{C}_{5} * \mathrm{C}_{7}
\end{array}
$$

But, for instance, the following are not species: $\mathrm{C}_{3} * \mathrm{C}_{4}, \mathrm{C}_{1} * \mathrm{C}_{7}, \mathrm{C}_{3} * \mathrm{C}_{5} * \mathrm{C}_{1}$, etc. 


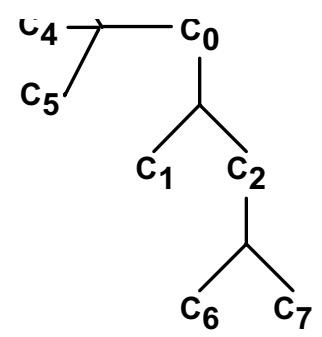

Figure 2: Specialization hierarchy.

We are interested in establishing the properties of species because in OASIS objects are always instances of species. In order to calculate these properties we will see some simplified case studies. Next we will analyze the equivalent in $D L$ of any static partition.

\subsection{Static partition hierarchies}

Given a specialization hierarchy with only static partitions the set of properties describing the behavior of any leaf class in the hierarchy is the union of properties from root class to the leaf class. In static and dynamic partitions of OASIS we have behavioral compatibility between superclasses and subclasses. Thus, the set of properties of any leaf class $\mathrm{C}_{\mathrm{i}}$ is given as follows:

i. Set of attributes:

$$
\operatorname{Atr}_{\mathrm{i}}=\cup \operatorname{Atr}_{\mathrm{k}} \quad \forall \mathrm{C}_{\mathrm{k}}=\mathrm{C}_{\mathrm{i}} \text { hasta } \mathrm{C}_{\mathrm{k}}=\mathrm{C}_{\text {root }}
$$

ii. Set of events:

$$
\mathrm{Ev}_{\mathrm{i}}=\mathrm{Ev}_{\mathrm{k}} \quad \forall \mathrm{C}_{\mathrm{k}}=\mathrm{C}_{\mathrm{i}} \text { hasta } \mathrm{C}_{\mathrm{k}}=\mathrm{C}_{\text {root }}
$$

iii. Valuation formulae in subclasses can only modify emergent attributes ${ }^{7}$ (and also attributes without valuations in superclasses), thus the set of valuation formulae is the union of valuations from leaf class to root class (i.e. the $D L$ formulae union).

iv. If a prohibition formula is modified in the subclass then the new condition must imply the superclass condition, thus the set of properties is the union of the properties from leaf class to root class (i.e. the $D L$ formulae union). In this way, only are considered the most specific preconditions.

v. Obligation formulae are established in the same way as prohibition formulae.

After deriving both structural and behavioral properties from every leaf class, it is immediate to calculate the properties of species.

Definition 2 Template of a species $\left(C_{0} * \ldots * C_{n}\right)$ is a class template with the following elements:

$$
\operatorname{Atr}_{0} \cup \ldots \cup A \operatorname{tr}_{n} \cup A t r_{e}
$$

7 That is, attributes defined in the subclass specification. 


$$
E v_{0} \cup \ldots \cup E v_{n} \cup E v_{e}
$$

$D L F o r m, \cup \ldots \cup D L F_{0 r m} \cup D L_{F o r m}$

where $A t r_{\mathrm{i}}, E v_{\mathrm{i}}$ and $D L F o r m_{i}$ are attributes, events and $D L$ formulae of class $C_{i} ; A_{t r}$, $E v_{e}$ and $D L F o r m e$ are the added attributes, events and $D L$ formulae being specified for the species $\left(C_{0} * \ldots * C_{n}\right)$. In case of overlapping of properties the calculus of properties follows the constraints previously mentioned.

\subsection{Dynamic partition hierarchies}

Whenever dynamic partitions are included, the process to determine properties of species is slightly different.

The idea is to translate all the subclass properties in every dynamic partition into an ascending direction. Figure 3 shows an example in which class $C_{2}$ has been dynamically partitioned. Thus we want to extend the dynamic formulae of class $C_{2}$ of Figure 3 separating the formulae due to $C_{6}$ from those due to $C_{7}$. Next specific considerations when deriving the $D L$ formulae set are described here.

\section{Migration by action occurrence}

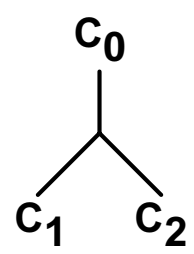

\section{$\mathrm{C}_{6} \quad \mathrm{C}_{\mathbf{7}}$}

Figure 3: Dynamic partition.

Given the dynamic partition $\left\{C_{1}, \ldots, C_{n}\right\}$ of the class $C_{j}$, then the template of $C_{j}$ including properties of subclasses for each class $C_{i}$ belonging to the partition, is given as:

i. $\quad$ Set of attributes: $A t r=A \operatorname{Ar}_{j} \cup A \operatorname{At}_{l} \cup \ldots \cup \operatorname{Atr}_{n}$.

ii. Set of events: $E v=E v_{j} \cup E v_{l} \cup \ldots \cup E v_{n}$.

iii. The set of valuations formulae is the union of valuation formulae of classes $C_{j}$ and $C_{l}$ to $C_{n}$. Each valuation must have a condition that controls when it is used.

iv. The set of prohibition formulae is the union of prohibition formulae from the classes $C_{j}$ and $C_{l}$ to $C_{n}$. Taking into account the behavior compatibility restriction, if $C_{i}$ redefines a prohibition of $C_{j}$ then the condition of $C_{i}$ prevails.

v. Obligation formulae are established in the same way as prohibition formulae.

vi. Migration processes defined in partitions will be included in class $C_{j}$ as a protocol containing valid action sequences. Prohibition formulae are directly incorporated into the set of prohibition formulae of $C_{j}$. However the behavior 
property of the class $C_{i}$ must add an occurrence predicate that evaluates the current state of the process. Thus, this such a protocol will be translated into prohibition formulae and valuation formulae in the same way as protocols.

vii. Repeat the procedure from i) to vi) for each dynamic partition of $C_{j}$.

Example 6 Given the following dynamic partition in OASIS:

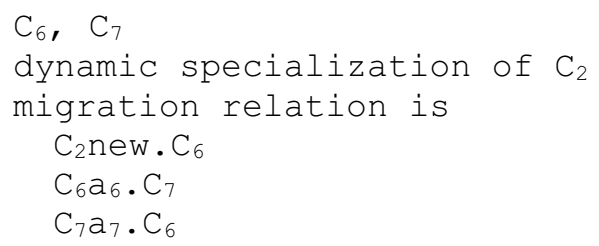

where we also have the following valuation formulae:

$$
\begin{array}{ll}
\Phi_{2} \rightarrow\left[\mathrm{a}_{2}\right] \Psi_{2} & \text { in class } C_{2} \\
\Phi_{6} \rightarrow\left[\mathrm{a}_{6}\right] \Psi_{6} & \text { in class } C_{6} \\
\Phi_{7} \rightarrow\left[\mathrm{a}_{7}\right] \Psi_{7} & \text { in class } C_{7}
\end{array}
$$

then, when translating these properties into the class $C_{2}$, the previous formulae are substituted by the following ones ${ }^{8}$ :

$$
\begin{aligned}
& \Phi_{2} \rightarrow\left[\mathrm{a}_{2}\right] \Psi_{2} \\
& \left(\left(\mathrm{p}=\mathrm{C}_{6}\right) \wedge \Phi_{6}\right) \rightarrow\left[\mathrm{a}_{6}\right] \Psi_{6} \\
& \left(\left(\mathrm{p}=\mathrm{C}_{7}\right) \wedge \Phi_{7}\right) \rightarrow\left[\mathrm{a}_{7}\right] \Psi_{7}
\end{aligned}
$$

and the following formulae are included:

$$
\begin{aligned}
& \left(\mathrm{p}=\mathrm{C}_{6}\right) \rightarrow\left[\mathrm{a}_{6}\right]\left(\mathrm{p}=\mathrm{C}_{7}\right) \\
& \left(\mathrm{p}=\mathrm{C}_{7}\right) \rightarrow\left[\mathrm{a}_{7}\right]\left(\mathrm{p}=\mathrm{C}_{6}\right) \\
& \neg\left(\mathrm{p}=\mathrm{C}_{6}\right) \rightarrow\left[\mathrm{a}_{6}\right] \text { false } \\
& \neg\left(\mathrm{p}=\mathrm{C}_{7}\right) \rightarrow\left[\mathrm{a}_{7}\right] \text { false }
\end{aligned}
$$

\section{Migration by attribute value}

In this case $D L$ formulae are now defined as follows:

i. $\quad$ Set of attributes: $A t r=A t r_{j} \cup A t r_{l} \cup \ldots \cup A t r_{n}$.

ii. Set of events: $E v=E v_{j} \cup E v_{l} \cup \ldots \cup E v_{n}$. Taking into account that an emergent event of a subclass must not be available if the object is not instance of that subclass, then it is necessary to add a $D L$ formulae that disable the occurrence of that event (see next example).

iii. The set of valuation formulae is the union of valuation formulae of classes $C_{j}$ and $C_{l}$ to $C_{n}$.

\footnotetext{
${ }^{8}$ Where $\mathrm{p}$ is a variable representing the current state of the migration process. Thus $\mathrm{p}$ is modified by valuation formulae.
} 
iv. The set of prohibition formulae is the union of prohibition formulae of classes $C_{j}$ and $C_{l}$ to $C_{n}$. Taking into account the behavior compatibility restriction, if $C_{i}$ redefines a prohibition of $C_{j}$ then the condition of $C_{i}$ prevails.

v. Obligation formulae are established in the same way as prohibition formulae.

vi. Each formula obtained from $C_{i}$ must add an occurrence predicate referring to the current state of the object.

vii. Repeat from i) to vi) for each dynamic partition of $C_{j}$.

Given the following dynamic partition in OASIS:

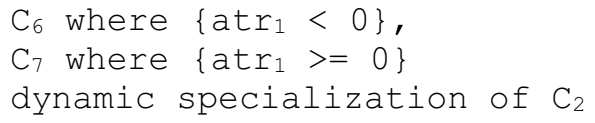

where we also have the following valuation formulae:

$$
\begin{array}{ll}
\Phi_{2} \rightarrow\left[\mathrm{a}_{2}\right] \Psi_{2} & \text { in class } C_{2} \\
\Phi_{6} \rightarrow\left[\mathrm{a}_{6}\right] \Psi_{6} & \text { in class } C_{6} \\
\Phi_{7} \rightarrow\left[\mathrm{a}_{7}\right] \Psi_{7} & \text { in class } C_{7}
\end{array}
$$

then, when translating these properties into the class $\mathrm{C}_{2}$, the previous formulae are substituted by the following ones:

$$
\begin{aligned}
& \Phi_{2} \rightarrow\left[\mathrm{a}_{2}\right] \Psi_{2} \\
& \left(\left(\operatorname{atr}_{1}<0\right) \wedge \Phi_{6}\right) \rightarrow\left[\mathrm{a}_{6}\right] \Psi_{6} \\
& \left(\left(\operatorname{atr}_{1}>=0\right) \wedge \Phi_{7}\right) \rightarrow\left[\mathrm{a}_{7}\right] \Psi_{7}
\end{aligned}
$$

and the following formulae are included:

$$
\begin{aligned}
& \neg\left(\operatorname{atr}_{1}<0\right) \rightarrow\left[a_{6}\right] \text { false } \\
& \neg\left(\operatorname{atr}_{1}>=0\right) \rightarrow\left[a_{7}\right] \text { false }
\end{aligned}
$$

\subsection{Translation process summary}

The translation process from class specification with specialization to $D L$ formulae is summarized in the following steps:

- The translation process is applied for each specialization hierarchy.

- The translation process in dynamic partitions (if they exist) starts from the bottom level. All properties defined in dynamic subclasses are added to the superclass. The translation process continues until reaching a static partition or until reaching the top class in the hierarchy (in this case, the translation process finishes).

- When all dynamic partitions have been substituted the properties for each leaf class containing only static partitions are determined.

- Eventually, properties for species can be determined from leaf class properties. This hierarchy flattening in separate species is the final representation. Thus, all species will be independent classes at the same level and without specialization relationships.

\subsection{Animation support}


From an ideal point of view the representation in $D L$ formulae finishes when all properties of species are specified. However, we also have to give a suitable support for taking into account the existing polymorphism in our OASIS model. Thus, an action in OASIS may reference a server class not being a species. In this situation the system must determine the full properties at the lower level. This implies a separation in two levels in our animation environment. On the one hand we have objects whose behavior is given by one species in which they were created. On the other hand we also have a class representing each original hierarchy class. In this additional class we keep a reference for each object belonging to it ${ }^{9}$.

Figure 4 shows how every class is considered at the same level (without hierarchy) and objects are associated to species. However, there are mappings from each class to their object instances. For example obj $j_{1}$ is an instance of class $\mathrm{A}$ and $\mathrm{B}$ and it is also instance of all species like $\mathrm{B} *$, etc.

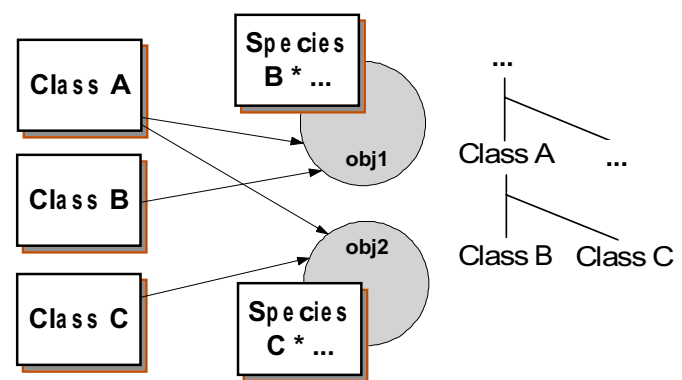

Figure 4: Representation example.

\section{Conclusion}

$O A S I S$ is a formal approach for the specification of object oriented conceptual models. In OASIS conceptual schemes of information systems are represented as societies of interacting concurrent objects. Animating such models in order to validate the specification of information systems is a topic of interest in requirements engineering.

Using inheritance we can specialize (or generalize) properties defined in classes. Specialization is an important modeling mechanism. There are two kinds of specializations in OASIS: by defining static and dynamic partitions. Objects in static partitions belong to a given specialized class during their whole lifetime. Besides, objects in dynamic partitions can migrate from one subclass to another one. The migration between subclasses of the same partition may be due to action occurrence or change in attribute values. A process migration represents the possible transitions among subclasses that an object can do. In addition, class templates of $O A S I S$ can be expressed as a set of $D L$ formulae.

Static and dynamic specialization constructs in conceptual modeling allow expressing directly some patterns. For example, in [2] the dynamic classification is implemented

9 A mapping between identifying mechanisms andids. 
using the pattern named state and the role mechanism may be implemented using the pattern named role object.

This paper gives the required steps to translate static and dynamic partitions in the same framework of $D L$ used for class template. Thus, an OASIS specification with specialization can be interpreted automatically as an equivalent specification without specialization in our $D L$-based framework. In this way, the current animation environment, working with valuation, prohibition and obligation formulae, can be used to animate specifications with specialization.

We have built a translator program to obtain a prototype from $O A S I S$ specifications automatically. The translator should be extended in order to include the mappings established in this paper. This work is being integrated into a CASE tool for system modeling supporting the OASIS approach and the methodology OO-METHOD [11] defined for $O A S I S$.

\section{References}

[1] Feenstra R.B. and Wieringa R.J. Validating Specifications of Dynamic Systems using Automated Reasoning Techniques. In J.C. Bioch and Y.-H. Tan (Eds.), Proceedings of the Seventh Dutch Conference on Artificial Intelligence, NAIC'95, pages 105-114, 1995.

[2] Gamma E., Helm R., Johnson R. and Vlissides J. Design Patterns: elements of Reusable Object Oriented Software. Professional Computing Series, Addison-Wesley, Reading, MA, 1994.

[3] Grau A. and Kowsari M. A. Validation System for Object-Oriented Specifications of Information Systems. In Manthey R. and Wolfengagen V. (Eds.), Proceedings of the First East-European Symposium on Advances in Databases and Information Systems (ADBIS'97), St. Petersburg, Electronic Workshops in Computing, Springer, September 2-5, 1997.

[4] Harel D. Dynamic Logic. In Handbook of Philosophical Logic II, editors D.M. Gabbay, F. Guenthner, pages 497-694, Reidel 1984.

[5] Grau A., Kuester Filipe J., Kowsari M., Eckstein S., Pinger R. and Ehrich H.-D. The TROLL Approach to Conceptual Modelling: Syntax, Semantics and Tools. In Ling T.W., Ram S. and Lee M.L. (Eds.) Proc. of the 17th Int. Conference on Conceptual Modeling (ER'98), Singapore, pages 277-290, Springer, LNCS 1507, November 16-19, 1998.

[6] Heymans P. The Albert II Specification Animator. Technical Report CREWS 97-13, Cooperative Requirements Engineering with Scenarios, http://sunsite.informatik.rwthaachen.de/CREWS/reports97.htm.

[7] Letelier P., Ramos I., Sánchez P. and Pastor Ó. OASIS 3.0: A Formal Approach to ObjectOriented Conceptual Modeling. Servicio de Publicaciones de la Universidad Politécnica de Valencia (SPUPV-98.4011), ISBN 84-7721-663-0, 1998. (in Spanish)

[8] Letelier P., Sánchez P. and Ramos I. Prototyping a requirements specification through an automatically generated concurrent logic program. Gupta (Ed.) Practical Aspects of Declarative Languages, Lecture Notes in Computer Science LNCS 1551, pages 31-45, Springer-Verlag, 1998.

[9] Letelier P., Sánchez P. and Ramos I. Process Specification for Objects interpreted into Dynamic Logic. III Jornadas de Ingeniería de Software (JIS'98), Murcia, pages 281-292, 1998. (in Spanish)

[10] Pastor O. and Ramos I. OASIS versión2(2.2): A Class-Definition Language to Model Information Systems Using an Object-Oriented Approach. Servicio de Publicaciones Universidad Politécnica de Valencia, SPUPV-95.788, 1995.

[11] Pastor O., Insfrán E., Pelechano V., Romero J., and Merseguer J. OO-METHOD: An OO Software Production Environment Combining Conventional and Formal Methods. Proceedings of Conference on Advanced Information Systems Engineering, CAiSE 197, pages 145-158, Barcelona, 1997.

[12] Meyer J.-J.Ch. A different approach to deontic logic: Deontic logic viewed as a variant of dynamic logic. In Notre Dame Journal of Formal Logic, vol.29, pages 109-136, 1988. 
[13] OBLOG Software S.A. The OBLOG Software Development Approach. (White Paper). 1999, http: / / www.oblog.pt/Download/Documentation.exe

[14] Rolland C., Ben Achour C., Cauvet C., Ralyté J., Sutcliffe A., Maiden N.A.M., Jarke M., Haumer P., Pohl K., Dubois E. and Heymans P. A Proposal for a Scenario Classification Framework. Technical Report CREWS 96-01, http://sunsite.informatik.rwthaachen.de/CREWS/reports96.htm.

[15] Sánchez P., Letelier P. and Ramos I. Constructs for prototyping information systems with object Petri Nets. Proceeding of the IEEE International Conference on SMC'97, pages 4260-4265, Orlando, 1997.

[16] Siddiqi J., Morrey I.C., Roast C.R. and Ozcan M.B. Towards quality requirements via animated formal specifications. Annals of Software Engineering, n.3, 1997.

[17] Taivalsaari A. On the Notion of Inheritance. ACM Computing Surveys, Vol. 28, N. 3, September 1996.

[18] Wieringa R., Jonge W. and Spruit P. Roles and dynamic subclasses: a modal logic approach. Vrije U., The Netherlands, 1995.

\section{A An example}

In this example a vending machine ( $\mathrm{vm}$ ) accepts coins (coin_in) increasing the customer credit (credit). This credit decreases (coin_out) when a product is given (give). The machine also allows canceling the operation (CANCEL) and then returning all the stored coins. The machine has a warning light (light_empty) that is switched on if the action switch_on occurs. The OASIS 3.0 specification of vm is the following:

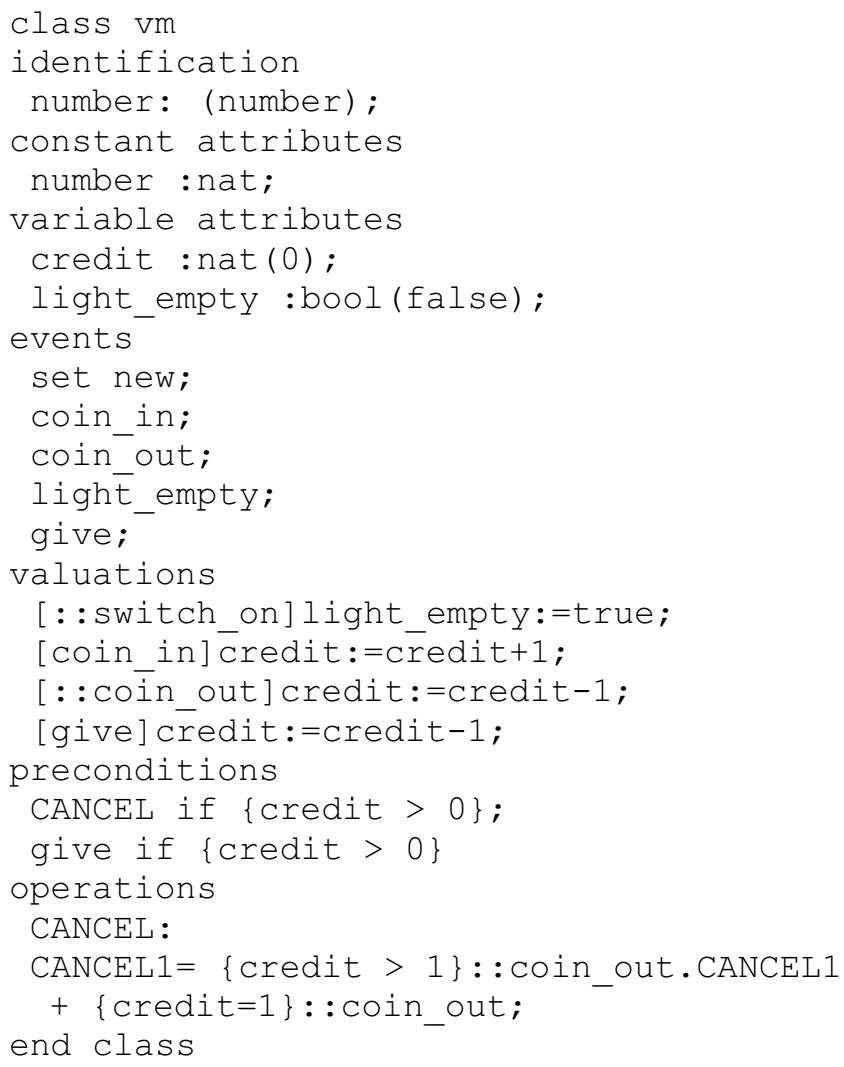

This machine is specialized in chocolate machines (choc_vm) and others (other_vm). This is a static partitioning, thus the OASIS specification is the following:

choc_vm, other_vm

static specialization of $\mathrm{vm}$; 
An object belonging to the class choc_vm has chocolates as products and their quantity is num_chocs. This quantity is reduced by one unit when giving a chocolate (give). It is not possible (a prohibition) to obtain a chocolate if there is neither credit nor chocolate. In $O A S I S$ we have:

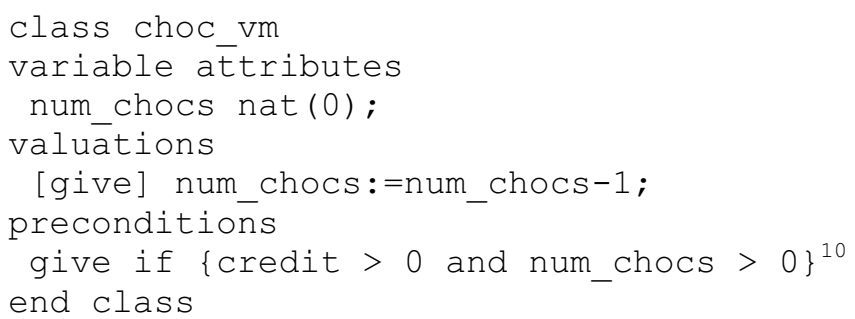

We will now consider a dynamic partitioning. The idea is to make a distinction between chocolate machines with products (with_choc) and without products (no_choc). A machine of type no_choc must switch on the warning (light_empty). In OASIS we have:

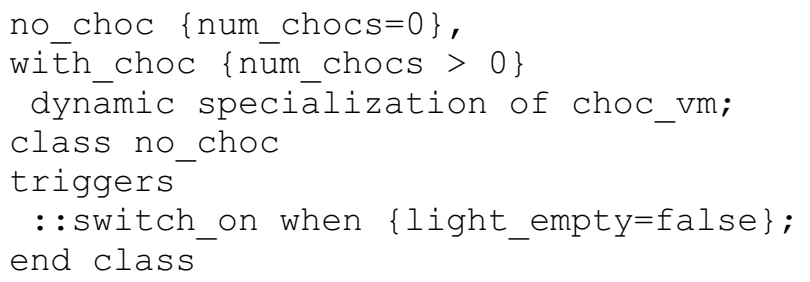

We will also include a static partition of class choc_vm. Thus we make a distinction between bounded ${ }^{11}$ (bounded_choc) and unbounded (unbounded_choc). The following $O A S I S$ specification gives the detailed information of this partition:

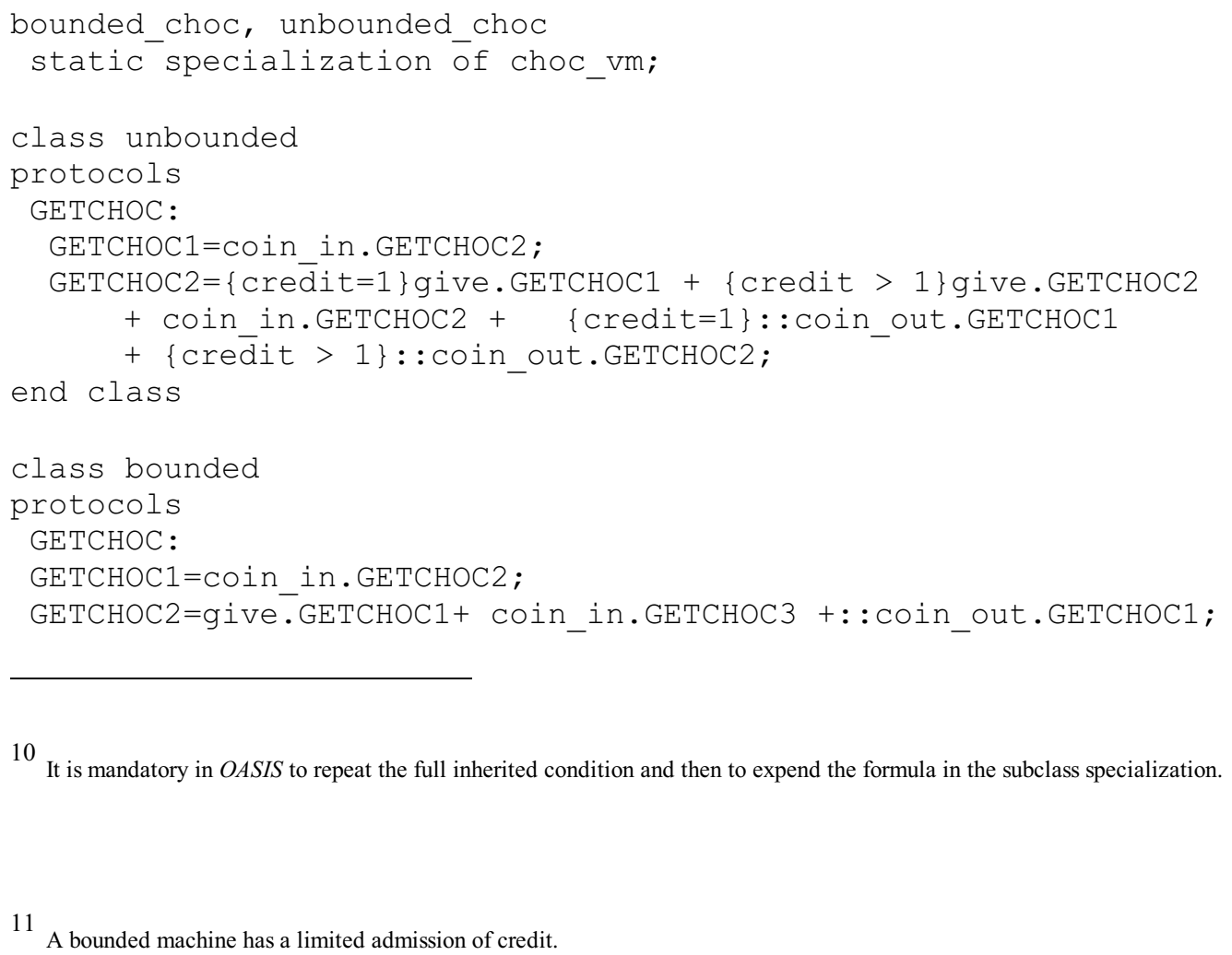


GETCHOC3=give.GETCHOC2+ coin_in.GETCHOC4 + : coin_out.GETCHOC2;

GETCHOC4=give.GETCHOC3 + : : coin_out.GETCHOC3;

end class

Eventually, vm is dynamically partitioned in working and broken_down. After the creation action (set) the machine starts in the working state. A machine may be repaired (repair) to reach the working state. When the action break_down occurs, the machines moves to the state broken_down where the events cancel, coin_in and give are not habilitated. The specification in OASIS (see the Figure 5) is the following one:
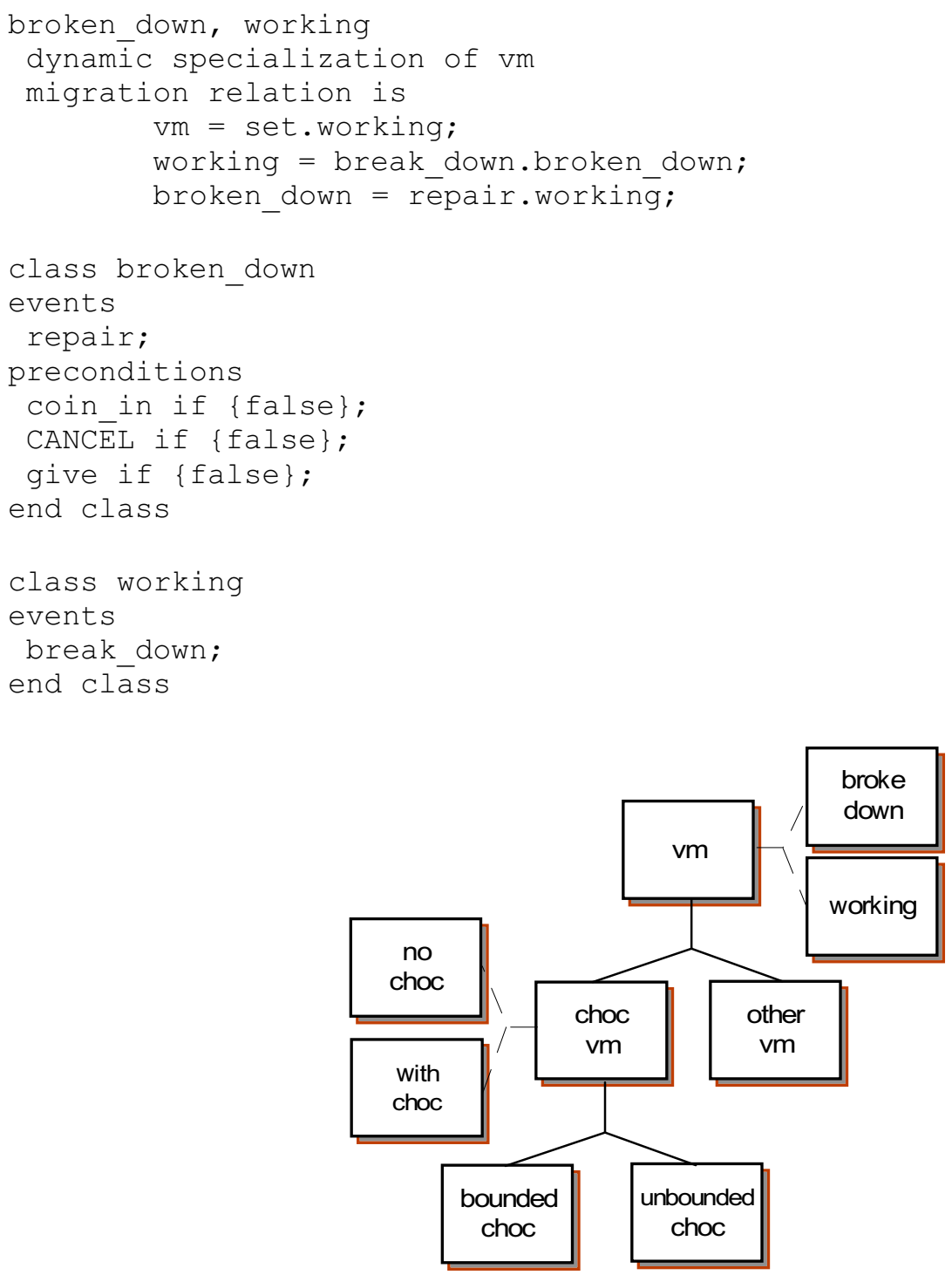

Figure 5: Specialization hierarchy.

\section{A.1 $D L$ for each $O A S I S$ class}

We give here a $D L$ formulae set for each class mentioned above:

\section{class vm}

credit $=\mathrm{N} \rightarrow$ [coin_in] credit $=\mathrm{N}+1$

credit $=\mathrm{N} \rightarrow[:$ :coin_out $]$ credit $=\mathrm{N}-1$ 


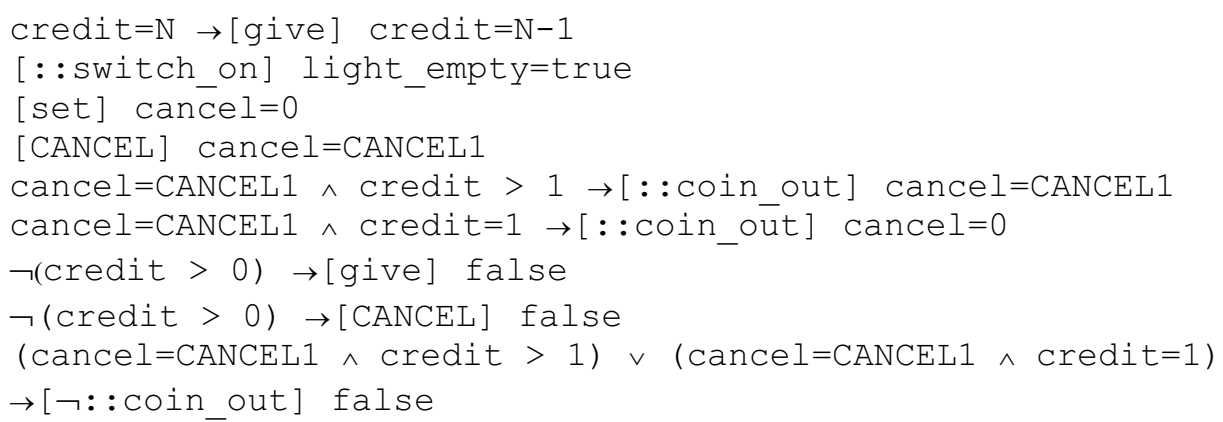

\section{$\underline{\text { class choc vm }}$}

num chocs $=\mathrm{N} \rightarrow[$ give] num chocs $=\mathrm{N}-1$

$\neg($ num_chocs $>0 \wedge$ credit $>0) \rightarrow$ [give] false

\section{class bounded choc}

[set ] getchoc=GETCHOC 1

getchoc=GETCHOC1 $\rightarrow$ [coin_in] getchoc $=$ GETCHOC2

getchoc $=$ GETCHOC $2 \rightarrow\left[\right.$ coin $^{-}$in $]$getchoc $=$GETCHOC 3

getchoc $=$ GETCHOC $3 \rightarrow[$ coin - in $]$ get $c h o c=$ GETCHOC 4

getchoc $=$ GETCHOC $2 \rightarrow[\mathrm{choc}]$ get $\mathrm{choc}=\mathrm{GETCHOC} 1$

getchoc $=$ GETCHOC3 $\rightarrow[\mathrm{choc}]$ get $\mathrm{choc}=\mathrm{GETCHOC} 2$

getchoc $=$ GETCHOC $4 \rightarrow[$ choc $]$ getchoc $=\mathrm{GETCHOC} 3$

getchoc=GETCHOC2 $\rightarrow[:$ : coin_out $]$ getchoc=GETCHOC 1

getchoc=GETCHOC3 $\rightarrow[:$ : coin_out $]$ getchoc=GETCHOC2

get $c h o c=$ GETCHOC $4 \rightarrow[::$ coin_out $]$ get $c h o c=$ GETCHOC 3

$\neg($ (getchoc $=$ GETCHOC 1$) \vee($ get $\mathrm{choc}=\mathrm{GETCHOC} 2)$

$\checkmark($ getchoc $=$ GETCHOC 3$)) \rightarrow[$ coin_in $]$ false

$\neg(($ getchoc $=$ GETCHOC 2$) \vee($ getchoc $=$ GETCHOC 3$) \vee($ getchoc $=$ GETCHOC 4$)) \rightarrow[$ choc $]$

false

$\neg($ getchoc $=$ GETCHOC 2$) \vee($ get $c h O c=$ GETCHOC 3$)$

$\vee($ getchoc $=$ GETCHOC 4$)) \rightarrow[:$ :coin_out $]$ false

\section{class unbounded choc}

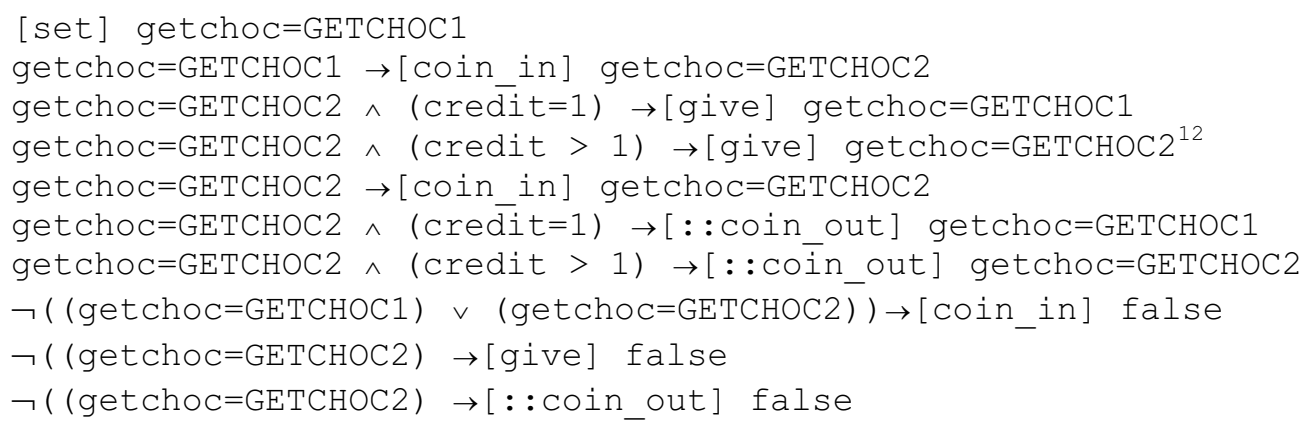

\section{class no choc}

light_empty=false $\rightarrow[\neg:$ :switch_on $]$ false 


\section{class broken down}

$\neg$ false $\rightarrow$ [coin_in] false
$\neg$ (false) $\rightarrow$ [CANCEL] false
$\neg$ (false) $\rightarrow$ [give] false

\section{A.2 Representation of dynamic partitions}

As mentioned above, properties of dynamic partitions need to be included in the partitioned class. The properties of the class choc_vm after incorporating the properties of classes no_choc and with_choc are the following ones:

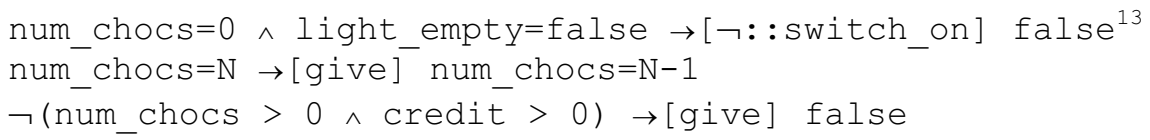

The inclusion of properties of the subclasses broke_down and working into the class vm plus the migratory process, gives the following set of $D L$ formulae:

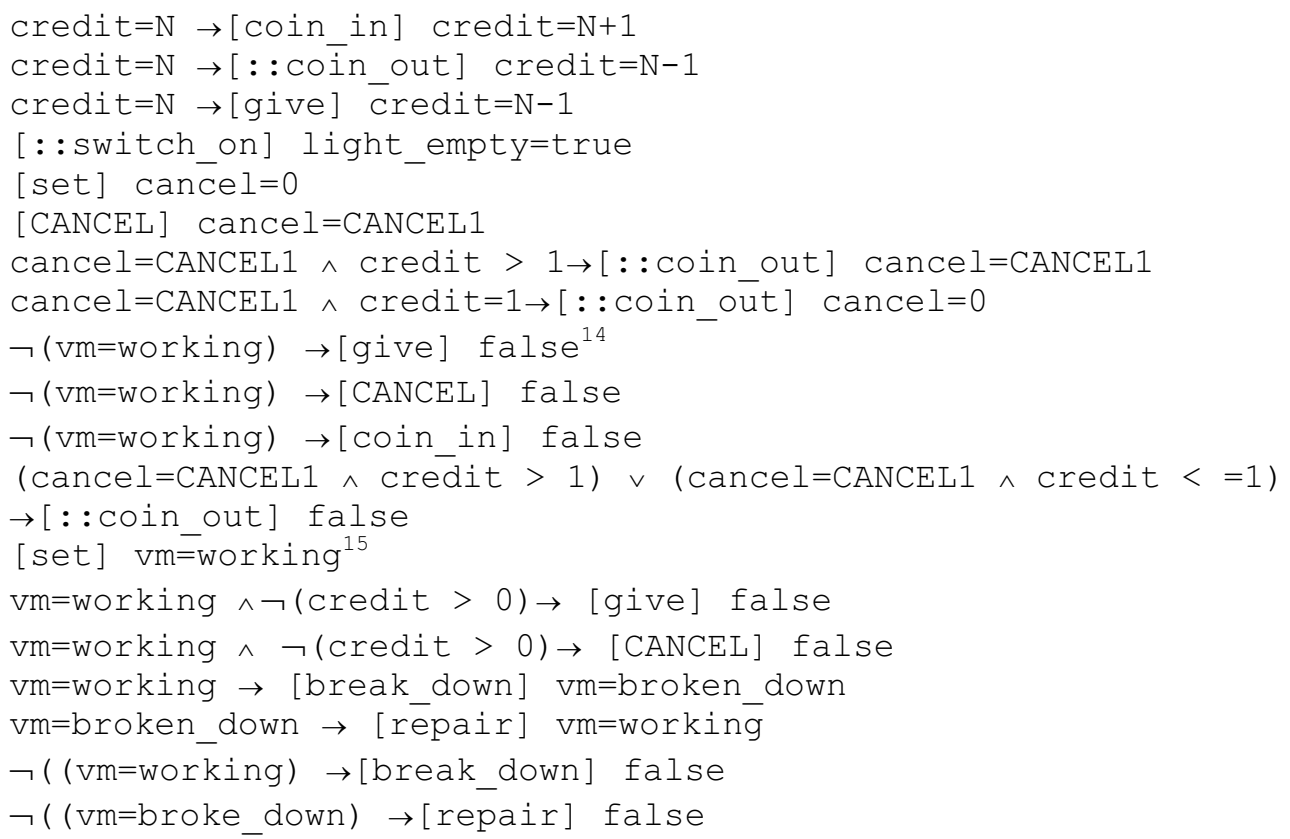

\section{A.3 Representation of static partitions}

After dealing with dynamic partitions we need to derive the $D L$ formulae for each path from each leaf class to the top class.

13 This formula is moved here from the dynamic subclass.

14 This permission and the next two ones replace the prohibitions ofm due to be more restrictive.

15 This formula and the following one are related to the migratory process of the dynamic partition 


\section{class vm - choc vm - bounded}

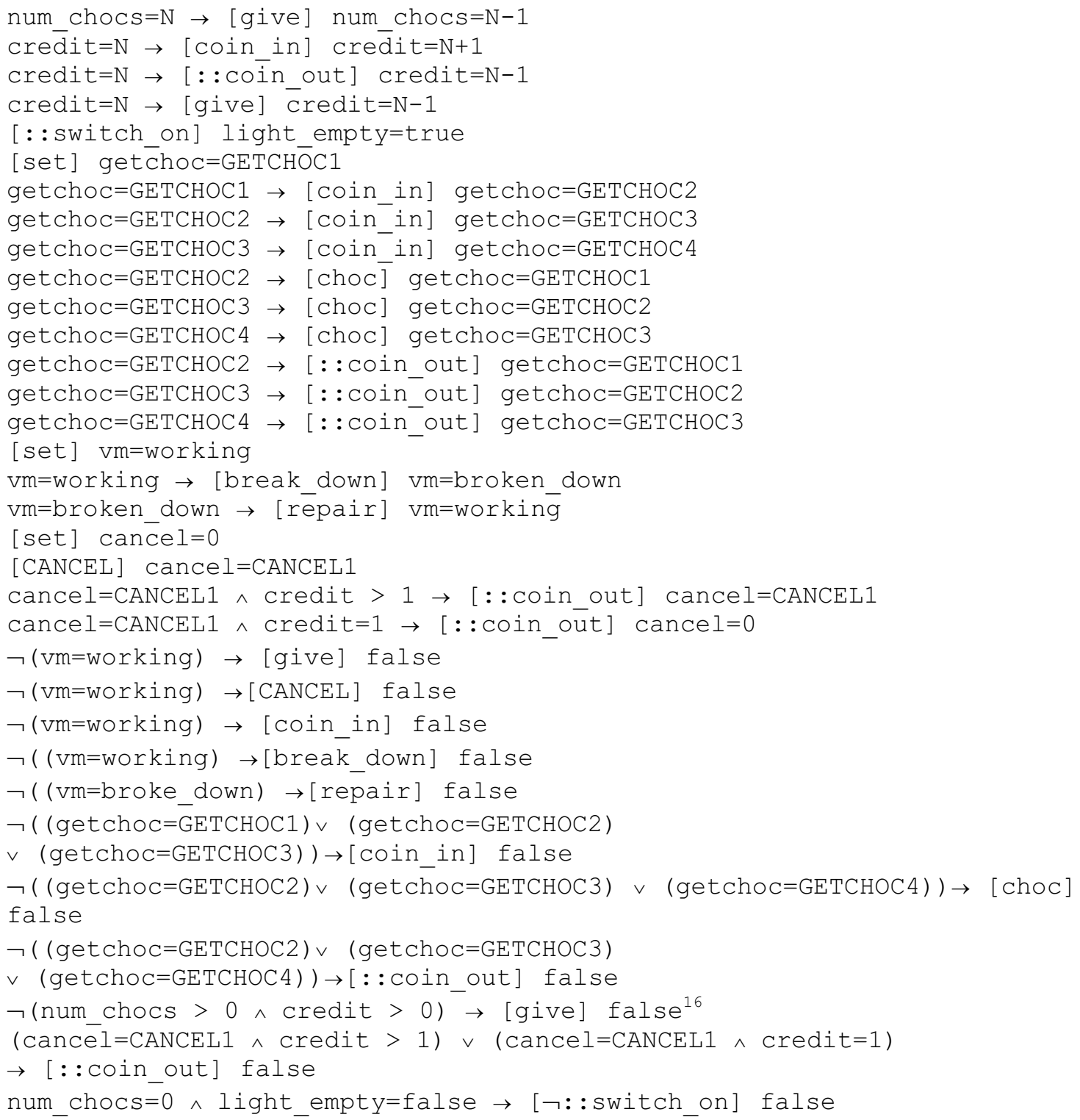

\section{class vm - choc vm - unbounded}

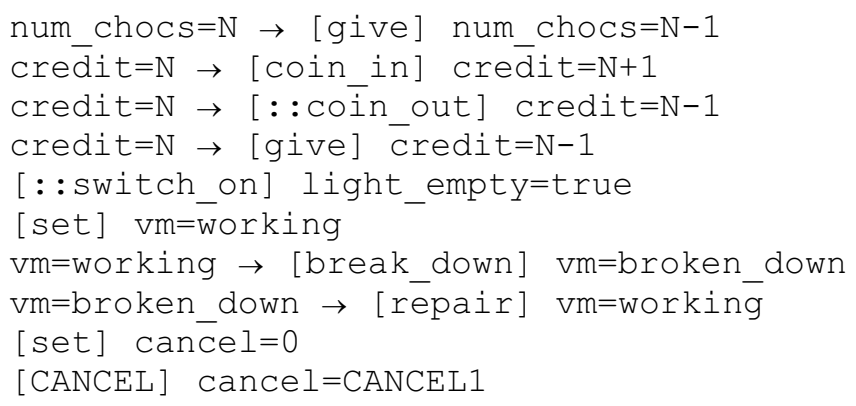

16

This prohibition formulae associated to get event what have just been mentioned just above can be mixed into one formula by using the logic connector ' $v$ '. 


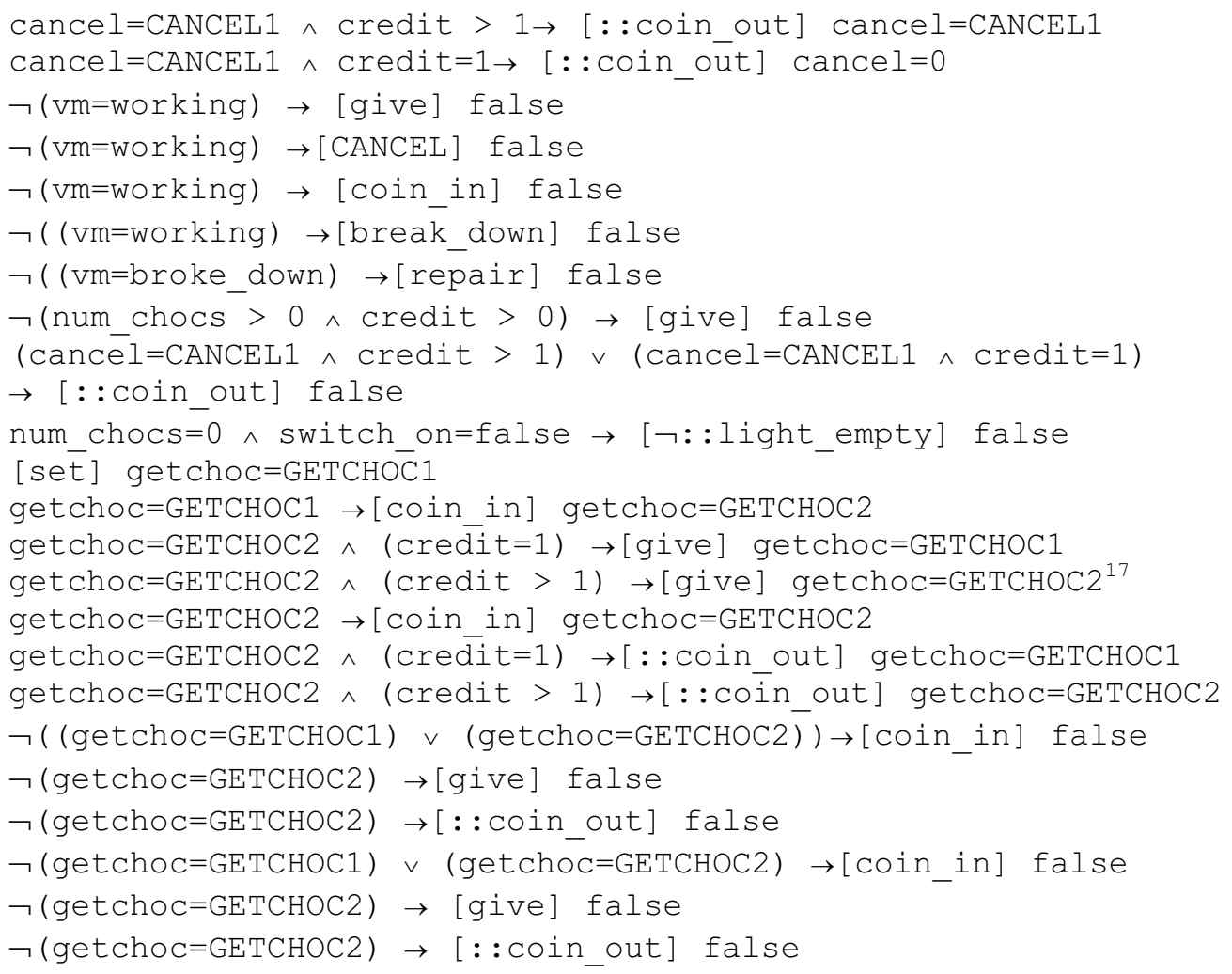

\section{class vm - other vm}

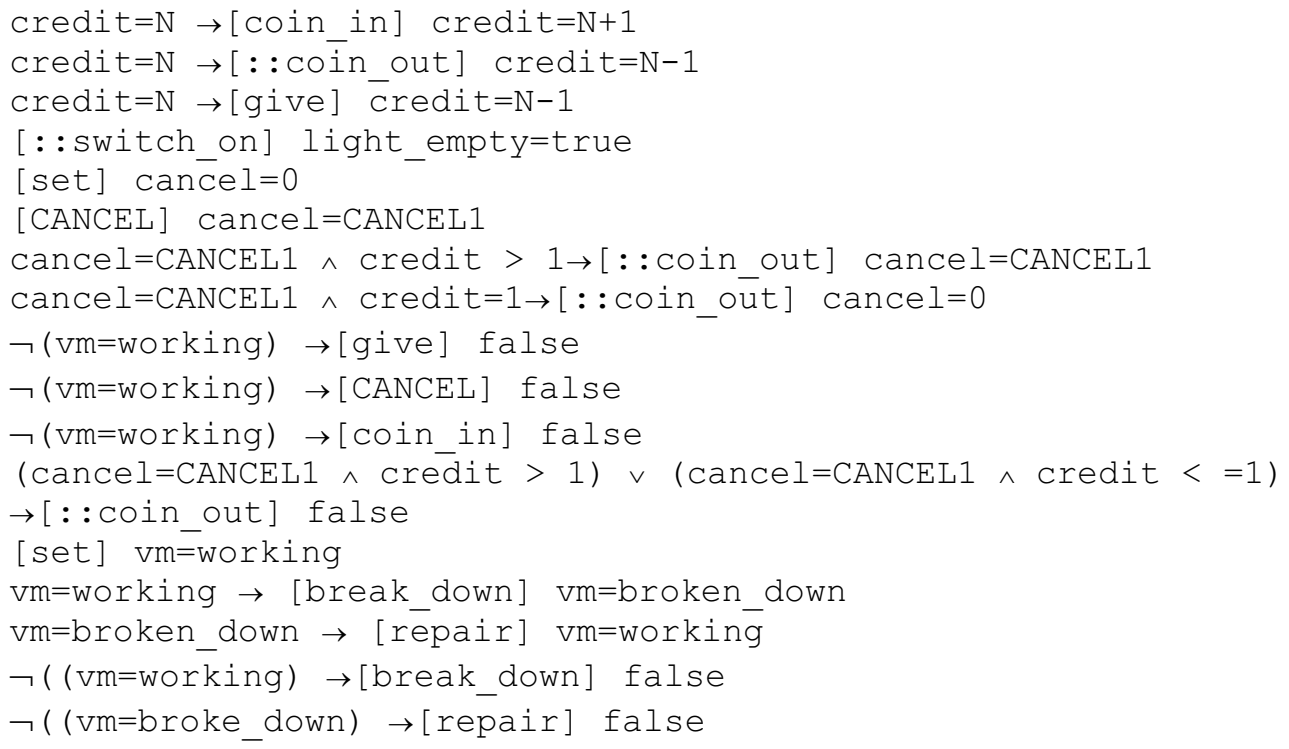

\section{A.4 Properties of species}


As mentioned above, it is possible to deduce the properties (as $D L$ formulae) for each species as the union of the respective properties. In the example we distinguish the following species involving only static partitions ${ }^{18}$ :

other vm

bounded choc

unboundēed_choc

Theses species correspond with each class mentioned above. In order to complete the calculus of properties we present here the set of identification mechanisms, attributes and events deduced from the specialization hierarchy of our example.

\section{A.4.1 Identification mechanisms}

The unique available identification mechanism (number) has been defined in the class $\mathrm{vm}$. This mechanism is inherited by every species.

\section{A.4.2 Attributes and events}

We present the attribute and event sets for each species. We assume that the possible conflicts caused by coincidental names have been previously adjusted as a part of the consistency verification process of the specification.

\section{$\underline{\text { Specie vm - choc vm - bounded }}$}

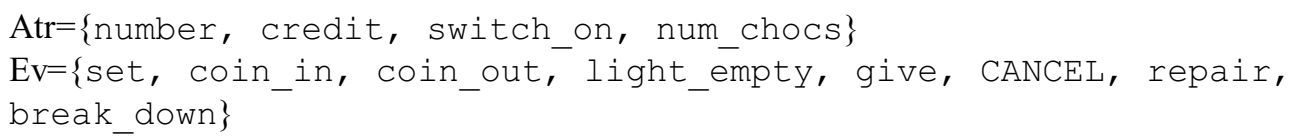

\section{$\underline{\text { Specie vm - choc vm - unbounded }}$}

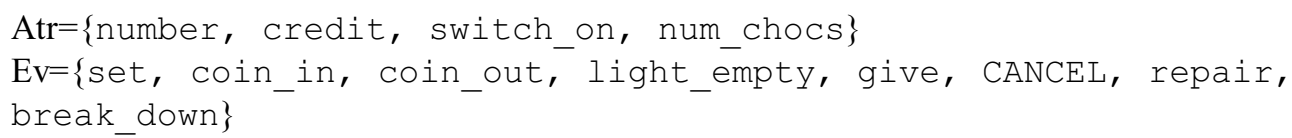

\section{Especie vm - other vm}

Atr $=\{$ number, credit, switch_on $\}$

$\mathrm{Ev}=\{$ set, coin_in, coin_out, light_empty, give, CANCEL, repair, break_down

\footnotetext{
18 In this example, taking into account that there is only one hierarchy and that there is only one double static partition of the same class, species are formed by only one class. In a more general case it should be necessary to connect the properties of each class.
} 